\title{
5.
}

\section{A Research-Based Approach to Game Writing Pedagogy}

Seth Andrew Hudson, PhD

Transactions of the Digital Games Research Association November 2021, Vol. 5 No 3, pp. 91-116. ISSN 2328-9422

(C) The text of this work is licensed under a Creative Commons Attribution - NonCommercial -NonDerivative 4.0 License (http://creativecommons.org/licenses/by-ncnd/ 2.5/).

IMAGES: All images appearing in this work are property of the respective copyright owners, and are not released into the Creative Commons. The respective owners reserve all rights

\section{ABSTRACT}

This paper discusses the importance of, and presents a possible framework for, phenomenological research of game industry practice to enhance pedagogy in computer game design (CGD) education. Built around examples from one such study on the practices of game industry writers, the author provides background for the study in question, outlines the theoretical framework of the research design, and presents an overview of the findings. A discussion of possible impacts and further applications in other subdisciplines of game development follows. 
Keywords

game writing, rhetorical genre studies, activity theory, phenomenology, pedagogy, writing studies

\section{INTRODUCTION}

Collaboration between the game industry and CGD programs in the past has focused on workforce development (Ashton 2010). It can be difficult to develop pedagogies in creative fields, but, as Mayers (2005) suggests, engaging existing frameworks to theorize practice can help fields learn from each other. CGD programs' faculty, as educator-researchers investigating the phenomenon of game development in context, can leverage the lived experiences of practitioners to construct more effective course design and instruction. In doing so, researchers can build rich data that may be applied to future work, thus driving further sophistication of CGD as a field of study in higher education.

This paper addresses the potential of this approach to researchenhanced pedagogy by outlining and discussing one such study focused on the pedagogy of game writing. The paper starts by providing background on the research problem, outlining the issues with developing a pedagogy of game writing. Next, the author presents an overview of a two-year phenomenological study of industry game writers to identify possible applications of this pedagogy-focused research approach. The discussion that follows highlights important questions and opportunities resulting from the study data and other scholarship.

\section{Problem Statement / Background}

The primacy of industry skills in CGD programs should be reconsidered, recognizing a need for entry-level skills to enhance student employability, while also acknowledging the faculty's desire to enhance their students' capacity for creativity and 
innovation later in their careers (Ashton 2010). In the subdiscipline of game writing, this reconsideration is fraught with complications.

Harry Brown stated, "Game development studios still struggle to define the role of the videogame writer and, more broadly, to reconcile the tasks of game design and storytelling” (2008 3). While the roles and tasks of game writing and game writers are poorly defined, hundreds of established professionals thrive as game writers and narrative designers in the field. A common sentiment surfaces throughout the literature and pervades the industry: there is nothing else like game writing (Bateman 2007; Chandler 2007; Dansky 2007; DeMarle 2007; Heussner et al. 2015; Sheldon 2013). In terms of scholarship, the game writing community's attempts to define their own practices is somewhat limited.

\section{Experimenting with Conceptual Frameworks from Other Disciplines}

Remaining open to scholarship and methods in other established disciplines that may not seem relevant at first glance, offers a vital opportunity to explore more effective pedagogies of game design in higher education. Educator-researchers in CGD pedagogy would benefit from adopting Maxwell's (2013) interactive approach to qualitative research design, one that remains flexible in methods and data collection, to test ideas and develop theory rather than draw conclusions.

Calling on my experience and training as an English composition instructor, my first inclination was to seek out resources to serve as exemplars and inform my approach to course design. However, I soon found that extant texts meant for instructive use (Bateman 2007; Chandler 2007; Despain 2009; Dille \& Zuur Platten 2008; Heussner et al. 2015; Lebowitz \& Klug 2012; Sheldon 2013; Skolnick 2014) are based primarily on professional anecdotes. Traditional writing genres, ranging from composition to poetry, can be found in game writing, but rather than communicating 
a single author's vision to the audience, game writing serves to enhance the other elements of the player's experience (DeMarle 2007; Sheldon 2013). Lacking relevant theoretical underpinnings in pedagogy or writing research, these texts are of limited use to researchers or educators looking to develop and enhance pedagogy.

"There is nothing like game writing" captures the common sentiment of these texts. Wendy Despain (2009) frames her edited collection on writing for video game genres as an industry-veteran authors' alternative to "drowning our sorrows and crying in our beer” (p. xiv). Statements like this signify the limitations of relying on these trade press publications as a scholarly assessment of the field.

Starting an inquiry with an exploration of these industry-borne texts may seem overly simplistic to some researchers, but "any meaningful inquiry into games must take the realities of the industry into account” (Egenfeldt-Nielsen et al. 2015). The distance between understandings in these two spheres does not indicate a lack of sophistication on the part of the industry or of game writers. Rather, it is indicative of an opportunity for educator-researchers to engage with the field directly (Hudson 2018).

The search for a more effective pedagogy for game writing courses in higher education became the focus of my doctoral thesis, Approaching a Pedagogy of Game Writing (Hudson 2018), collecting data via semi-structured interviews with AAA game developers over the course of two years. What follows is a brief discussion of two such theories, prefaced with my bias stemming from a background of teaching composition. With such a vast corpus of scholarship, interested readers should seek out more from the sources referenced.

We do indeed stand at a frontier of interactive digital media, of games as a storytelling tool; fortunately for educators, colleagues 
in other fields have investigated how humans interact with one another in collaborative pursuits. Some of the theories that informed my research design, such as those first presented by Vygotsky (1978) and Miller (1984), may seem quite dated; many of the methods of data collection and analysis are well worn territory for researchers in other fields. The goal here is to expose readers to potential lines of inquiry, offering just one example of how common theories and methods from other disciplinary traditions can be repurposed to shed light on a relatively new field of study.

\section{CONCEPTUAL FRAMEWORK AND RESEARCH DESIGN}

While basic, the strongest advice for students wishing to pursue game writing is to demonstrate "not only that they can write, but they can write for games" (Dansky 2007). The goal of this study is to approach what it means to write for games, with the eventual goal of incorporating that knowledge into game writing pedagogy. According to DeVoss, Eidman-Aadahl, and Hicks (2010), pedagogical practices to support writers' development fall into three strands:

- Supporting students in the process of writing and working in a community of writers,

- Studying the craft of writing and how it functions across genres, and

- Helping students analyze the rhetorical situations where writing takes place to instill flexibility and strategic thinking when addressing new contexts.

The purpose of this study is to support these goals by exploring the game writing practitioners' lived experiences in terms of writing scholarship and research, and effectively apply these findings to pedagogy. 
Given the role of game writing within AAA game production, constantly changing from one project to the next and working under constraints dictated by technology and organizational structure (Bateman 2007; Chandler 2007; Dansky 2007; Despain 2009; Dille \& Zuur Platten 2008; Heussner et al. 2015; Sheldon 2013; Skolnick 2014), the game writers' experiences are most effectively analyzed as part of the complex system of game development. A synthesis of rhetorical genre studies (RGS) and cultural-historical activity theory (CHAT) ultimately drove my research design, as both approaches look at writing in the context of production.

\section{Rhetorical genre studies (RGS)}

Genre, as defined below, is inescapable in writing instruction, as it is part of the cultural context writers work within (Devitt 2000). Russell (2001) holds that effective writing instruction should focus on what instructors want students to do, rather than what they want them to know.

RGS approaches to writing embrace the dynamic nature of any working situation. The game writing literature presents the lack of standard formatting as a limitation to learning the practices of game writing, but rhetorical genre researchers understand that all writing is situational. This means that the common notion of, "there is nothing like game writing" found in the literature is a null point. Were the same logic informing this sentiment applied to writing in other professional situations, it would be true to say, "there is nothing like any writing" in specific professional settings.

Relying on John Swales (1990) for a working definition, this study categorized genre in the following ways:

- Genre is a class of communicative events playing a vital role in game writing that encompasses both written and oral communication;

- The principal feature of the communicative events from 
which genre evolves is a shared set of purposes among individuals within a particular professional setting;

- Exemplars of a particular genre vary in their prototypicality.

To this last point, Swales identified a definitional approach and a family-resemblance approach. The latter focuses on loosely shared interrelationships rather than a list of defining features. Swales' family resemblance approach to genre is useful in the context of the game industry, not only for writing but for analyzing the relationships between all the moving parts required in game production (Hudson 2018).

The industry-borne game writing literature presents the lack of standard formatting-lack of prototypicality-as a limitation to learning the practices of game writing, but RGS researchers understand that all writing is situational. Exploring the processes of game writing in context serves the scholarly purposes of this study, while also presenting useful information for the field of computer game design. Applying RGS as a frame to analyze the practices of game writers, and game developers in general, is a key tool in challenging the current assumptions in and about the field.

Phenomenological approaches can provide rich descriptions of the social contexts surrounding writing, but defining a genre also requires the textual inputs and outputs of the activity (Bazerman, 1997). Luckily, CGD students are likely very familiar with the outputs, games in this case. What they do not have access to are the inputs, the actual work performed by game writers in context. Analyzing genre should go beyond the features we are already aware of to identify the implicit practices - the functional interactions of writing and its creators-of those in the field (Bazerman \& Prior 2009).

Borrowing concepts from RGS-focused on the real-world contexts where texts are created (Bazerman \& Prior 2009; Devitt 2000; Miller 1984; Russell 2010; Swales 1990)—this research 
design allowed for meaningful investigation of game writers in their respective professional settings.

\section{Cultural historical activity theory (CHAT)}

CHAT, for instance, is conducive to defining the processes and the conditions for attaining concrete goals in a complex system, while also factoring in the ever-present dynamics of power, money, culture, and history (Foot 2014). Despite romantic notions of video games being developed in basements by small groups of talented, enthusiastic friends, "[i]t is important to consider the mass production of games and the industrial process that makes their production possible, since both their aesthetic form and their consumption are influenced by this overarching structure" (Egenfeldt-Nielsen et al. 2015).

Scholars and researchers continue to extend Vygotsky's (1978) model of activity theory —notably Engeström (1999), Foot (2014), and Nardi (1995) — but three central ideas remain at the core of what is now more commonly known as cultural historical activity theory:

- Humans act collectively and learn by doing, communicating in and through activities;

- Humans make, use, and adapt tools-literal and conceptual—-to learn and communicate; and

- The community is central to making and interpreting meaning in all forms of learning, communicating, and acting.

Given the complexity of game development, complicated by poorly-defined roles and a lack of standard practices (Newman 2013), CHAT provides a valuable framework for meaningful understanding of any role in game production. CHAT's attention to constraints within a system is also uniquely valuable for analyzing 
the work of game writers who are often tasked with executing others' concepts while accounting for interactivity (Hudson 2018).

It is important to note that this study's eventual focus on game writers in the AAA space was driven by the conceptual framework; CHAT, in particular. Many of the games that are leading the way for the medium's maturation via more sophisticated approaches to narrative have come from the indie games space; given the creative constraints present within any profit-driven industry, that trend will likely continue. Focusing on AAA game production and framing it as an activity system can follow the footsteps of other CHAT studies that seek to understand large, complex systems. There are a growing number of spaces where games and other interactive experiences flourish, but considering CHAT in research design illuminated other interesting lines of inquiry to explore tensions within the game industry.

In addition to insights that help inform pedagogy aimed at professional development, the CHAT framework often serves to highlight contradictions and tensions within the systems they investigate; those same tensions and contradictions indicate space for innovation across the entire system (Engeström 1999). Aside from my goal of developing more effective pedagogy, the incorporation of CHAT methods of data collection and analysis offers a unique chance to overcome what O’Donnell (2014) calls the "industry's pervasive secrecy" and attempts by the game industry and its developers to hold themselves "as distinct from other industries." One phenomenological study seeking to learn from the experience of industry practitioners is not likely to unveil this secrecy, but an accretion of research focused on the experiences of individuals within the system might do so over time.

CHAT studies often employ phenomenological interview methods, as these allow practitioners to make tacit knowledge explicit while affording the interviewer the new insights regarding their own role in the system. Educator-researchers engaging industry actors 
through research come away with unique insights as the observed phenomenon is seen through the lens of an educator. Used to better understand fields ranging from public healthcare systems to theater production, CHAT research aims to advance ways of thinking about professional practices, shaping or reshaping them in context, and often with the goal of developing related teaching strategies and curricula (Foot, 2014).

According to Bazerman and Prior (2009, 2), to view writing through the lens of RGS, "we need to explore the practices that people engage in to produce texts as well as the ways that writing practices gain their meanings and functions as dynamic elements of specific cultural settings." CHAT is equally useful when considering writing in game industry contexts, as it incorporates the complex relationships of power, money, culture, and technology (Foot 2014). By making these connections explicit, this study provides a clearer analysis of the game writing process and formulates pedagogical recommendations that can help students prepare to perform in that specific genre.

CHAT and RGS demonstrate the complexities in writing in various settings, so the thinking of these scholars allowed me to limit my scope and refine my methods to add something to the larger conversation about writing instruction and the game industry. Namely, I hope to provide a model for game writing that accounts for the realities of production, and develop an effective pedagogy for game writing - one that incorporates the realities of higher education and best practices in instruction.

\section{Research Design}

After reviewing relevant literature and settling on a conceptual framework combining RGS, CHAT, and my personal experiences as an educator, the ultimate design of the study was built on two deceptively simple research questions:

- What functional competencies are required of 

professional game writers?

- To what educational experiences do game writers attribute the development of these competencies?

Throughout the course of the study, I allowed my knowledge and experience as an educator to inform my efforts. While open to any theoretical direction the data suggests, my approach was pragmatic, seeking results conducive to applicability and action (Brinkmann \& Kvale 2014), rather than a purely phenomenological approach solely focused on representing experience. An approach of this kind attempts a deeper understanding of the meanings behind everyday experiences, while also offering plausible insights that allow others to understand those experiences more completely (van Manen 1990).

Following Prior's (2009) suggestion, these semi-structured interviews shifted between questions grounded in specific knowledge and scholarship, and questions that surfaced naturally during the conversation. Direct questions regarding the knowledge, skills, abilities, and characteristics of game writers fell into this category (e.g., "Tell me about your favorite writer to work with.”). I also asked more open-ended questions regarding the dayto-day activities of game writers (e.g., "Describe what it looks like when you sit down to write," eventually followed by, "How is your writing process different in the studio?”).

The steps taken to ensure anonymity-giving pseudonyms to participants, removing references to specific studios and game titles, and limiting specifics in demographic reporting given the close relationships within the game writing community-allowed participants to be honest and open in their responses. In cases where the interviewee responses began with, "Since this is off the record," it was apparent that providing participants anonymity allowed them to be more forthcoming, likely offering richer data. Note: All recruitment materials and informed consent documents were reviewed and approved by my institution. 


\section{Data Collection and Analysis}

Recruiting participants at industry events and through relevant email lists, one-on-one interviews were scheduled for mutual convenience. While I created a guide that outlined particular questions that were meant to assist me as an interviewer and give uniformity to in situ notes and coding, the interviews were conversational. This semi-structured approach to interviewing allows for co-creation of knowledge (Wengraf 2001) and provided space for me to interpret via my conceptual lens.

Honoring these interviews as conversations, rather than a collection of data to be scrutinized once transcribed, I was able to interact with participants in the process. Assisted by the interview guide, I coded responses in situ, both during and immediately after interviews. This allowed me to be present in the conversations without losing focus on the intention of the interview.

Working with the transcriptions after the fact, I performed open coding to identify emergent themes-sentiments that surfaced across interviews or seemed pertinent to my purpose. Another round of coding followed, reviewing the transcripts with audio to verify the appearance of those emergent themes while identifying any pointed responses from participants. Pointed responses were those sentiments imbued with emotional intensity, given importunate explicitly, or repeated multiple times throughout the interview. Interviews are conversations, not transcripts (Brinkmann \& Kvale 2014); making an effort to be present in the interviews and spending time with the audio after transcription was the key to collecting meaningful data in this study.

Now coded, I adopted aspects from Brinkmann and Kvale’s (2014) framework for interview analysis focused on adopting their use of meaning condensation tables to process the data. These condensation tables were produced after the first two rounds of coding by revisiting the interview transcripts and audio, and extracting the natural units that accurately portrayed the context of 
specific responses. In practice, this meant returning to the coded transcripts and audio with a focus on isolating the humanity around a particular statement.

The process of generating these natural units, repeated multiple times, looks like this: choose a specific instance coded in the transcript, find that portion of the interview in the audio recording, then rewind and playback the recording as needed to identify and capture the whole sentiment that produced the coded utterance in this question. Finally, the text from that natural unit is captured via the transcript and set aside. This curated sampling of natural units was the final data set used in analysis.

This engagement with the data was of particular use as an educator. Though it required many hours, weeks, and months to process and analyze, the time spent working with the data yielded immediate insights for my practice in the classroom. Even if I had failed to complete and publish the work, my students would benefit.

While this may seem daunting, this approach to data analysis deepened my understanding of the participants, the people, who kindly offered their time to assist me with my work. Given the purpose of enhancing game writing pedagogy, this phenomenological research approach was effective.

\section{Limitations}

This approach to data analysis did limit the sample size, curating a data set based on multiple interviews with seven different individuals. Each of the seven met the criteria developed in the first round of coding - I will not share it here to respect anonymity. Setting this limitation was indeed intentional, based on Moustakas' (1994) thinking that a purposeful sample can maximize the richness of the data. As Creswell (2006) advised, "An individual writing a phenomenology would be remiss to not include some discussion about the philosophical presuppositions of phenomenology along with the methods in this form of inquiry” 
(59). Basic philosophical stances on phenomenology hold that it describes the essence of the lived experience, rather than draw conclusions based on the data collected.

Outside of questioning the reliability of any particular accounts, each participant offered reflection. The reporting of each interviewee's lived experiences is specific to his or her career path, beliefs, biases, and a host of other factors informing who they are as people, in addition to their professional selves. Shared in the form of reflection, and sometimes relying on a participant's perception of others, at a minimum, the responses are filtered through hindsight. As opposed to ethnographic approaches that may provide more accurate data on day-to-day work through observation, this study relies solely on what the participants said about their work hindsight.

\section{OVERVIEW OF SIGNIFICANT FINDINGS}

Rather than identifying finite abilities, skills, and knowledge, data analysis revealed more malleable categories, termed 'areas of competence' necessary for game writing; these included: writing and storytelling, communication and collaboration, understanding systems and dynamics, tool proficiency, and understanding play. Beyond these areas of competence, the study also identified three essential roles of the game writer-wordsmith, sensemaker, and advocate - that may serve as a structure for examining how various areas of competence are engaged, alone or in combination, across the array of tasks performed by industry game writers.

\section{Areas of Competence}

Listed below, each area of competence encompasses a group of competencies required to support a productive career in game writing:

- Writing and storytelling-required to produce written text and generate engaging story content efficiently; 
- Communication and collaboration-required to work effectively with other individuals in a studio environment;

- Understanding systems and dynamics-required to perform functions at a high level within the limitations of production and technology;

- Tool proficiency-required to demonstrate the transfer of writing and storytelling skills to the tools, both technological and conceptual, of the industry; and

- Understanding play-required to create content for games by producing writing conducive to interactivity and allowing for player freedom.

Learning outcomes that promote the areas of competence outlined in this study are enhanced when framed in the three essential roles of wordsmith, sensemaker, and advocate.

\section{Essential Roles of the Game Writer}

This study identified three essential roles that game writers play, in some capacity, across contexts. Although little uniformity exists across titles and roles in the industry (Bates 2004; Newman 2013), these roles encompass the array of tasks the game writer may perform in any given setting. Summarized, they include:

- Wordsmith - the game writer's focus is on execution rather than creativity. Completing the assigned tasks of game writing requires flexibility when crafting with text.

- Sensemaker - the game writer seeks to understand the creative views of individuals in other subdisciplines to build a sense of ownership for the game's story from all those involved in the production.

- Advocate-the game writer champions the story vertically to the decisionmakers and horizontally across 
the subdiscipline teams, relying on emotional intelligence and careful observation.

Students can conceive of any given task in terms of hypothetical industry contexts - the rhetorical situation and its context within the activity system - while relating them to a combination of these essential roles.

\section{Application to Pedagogy}

My experience conducting this research has directly affected my pedagogy, but also offered a basis for designing curriculum. I present a small sample of these pedagogical applications and/or considerations regarding the areas of competence below.

Game Discipline Knowledge (writing and storytelling): while this study focused on game writing, the specific subdiscipline of game design I sought to better understand, this particular area of competence is interchangeable. Art, animation, audio, programming, etc. could easily stand in. In some cases, this knowledge can be attained from other disciplines at university. In the instance of game writing, a few courses on creative writing, regardless of genre. In the classroom, this means borrowing from my background as a student taking playwriting, screenwriting, and poetry courses.

Communication and collaboration: the prevalence toward collaborative team projects in CGD is valuable indeed, but maximizing the pedagogical value of these group interactions requires reflection. By giving students a task and letting them develop mediating tools as they see fit, or alternatively demanding strict adherence to a particular approach that reinforces attention to detail and accountability, a balance can be struck by introducing thoughtful reflection on the process of creation.

Understanding systems and dynamics: giving students an understanding of the hierarchical structures within game 
development offers a lens for situating even the smallest group project in the larger system of game development. Even on an indie team consisting of a handful of individuals, the relation of one task to another, while factoring in the technological challenges that accompany each task, is vital to success. This also factors in the hierarchical structure present in larger-scale production. In the classroom, roleplaying - and often times, playing with roles mid-project—affords students this understanding. Low stakes collaborative activities suit this purpose, making outrageous demands for writers to produce $X$ in the next 45 minutes then letting them know it needs to be $\mathrm{X}+\mathrm{Y}$ about 25 minutes into their work.

Tool proficiency: reinforcing that writing is about more than generating ideas; incorporating technological challenges alongside narrative content creation allows for greater creative growth. In the classroom, students can create content using Twine or Inklewriter. Often times I combine the creation of a user guide with these assignments, asking the students to consider how they might explain the use of these tools to others. If the availability of gamespecific software is not an option, giving students tasks with common tools such as Microsoft Word can have equal benefits. Challenging students to use these tools as more than word processors, engaging their creativity to use seemingly mundane software, and enhance the visual impact of a project, allows them to inject identity into documents. Also, in the case of game writing, teaching students to work within spreadsheets is a must.

Understanding play: a thorough understanding of play and players is an obvious requisite for anyone aspiring to make games. In my courses, which often include students outside the CGD major, this means finding a way to present player interaction that is valuable to the experienced and novice alike. Readings from game studies, recordings of post mortems, and actual play in the classroom offer a chance to examine prior experience with play and give it meaning. 
Due to the necessary collaboration between game writers, other subdisciplines (e.g., art, audio, programming), and player expectations of interactivity, the essential roles and areas of competence that emerged represent the differences between writing for games and writing in other mediums. The essential roles of wordsmith, sensemaker, and advocate are the frame for the course. I introduce this concept early and reinforce it throughout the course with explicit reminders - often times, students are asked to reflect on particular experiences in writing - so that students understand which roles they are playing in given situations. This deepens their understanding of the rhetorical situation in the context of larger, though sometimes hypothetical given our classroom setting, systems at work.

\section{Researcher Reflections}

The data collected in this study, much like extant texts on the subject, still focused on debunking the perceived myths about a writer's work in preparation for the practical realities of the field. According to Peery (2016), if there are rules for writing in the industry, they are largely developed in-house for specific projects. In university composition programs, however, the concept of writing as a loosely-defined set of interrelated tasks and processes is now common. Teaching writing with a focus on process, rather than product, is so widely accepted that "it may be difficult to imagine alternative instructional approaches" (De La Paz \& McCutchen 2011, 32).

Making tacit professional knowledge explicit is valuable to advancing any field (Schön 1983), but is particularly useful when exploring new fields with few standard practices. The real value comes in the educator-researcher's interpretation and eventual implementation into pedagogy. Capturing the game industry professionals' perceptions is a first step in challenging the assumption that "there is nothing like game writing." 
CGD instructors that conceive of game writing, or any subdiscipline, as a mere synthesis of relevant tasks common in the industry are not likely to produce anything more than a list of possible classroom activities. Those who are willing to experiment in the classroom and attempt new methods of instruction that challenge traditional notions of higher education can truly empower students (Hudson \& Willis 2019). Instructors who frame pedagogy in more abstract ways, such as these essential roles, are better able to develop effective methods of instruction regardless of perceived constraints of time or resources.

\section{DISCUSSION}

While a great number of words are devoted to my journey of approaching a more effective pedagogy of game writing, I hope educator-researchers in the field of CGD take away something more. The study presented above highlights the potential for collaboration between industry and academia that is driven by instructors-experienced professionals with a wealth of knowledge-and grounded in research. Rather than simply amending coursework per the views of the practitioners in the field, faculty that engage in research on the industry in concert with academic disciplinary knowledge and teaching expertise, develop more effective instruction and approaches to curriculum design.

Individual studios and professional organizations such as the International Game Developers Association have demonstrated a willingness to work with CGD programs, but "collaboration, dialog and attempts to bridge industry and higher education gaps seem to be focused principally around workforce development" (Ashton 2010, 44). While logical, that goal is more elusive than it may seem. Preparing students with industry-specific skills is difficult given the lack of uniform practices in the industry and the rapid pace of change driving the industry's evolution. 
Just as the medium "must move beyond computer science and art to simply code games and make them look good" (Salmond 2016, 24), CGD educators must seek more innovative approaches to training students-a greater focus on game storytelling is one way forward. Greater focus on narrative has the potential to speed the sophistication of the medium (Jacobs 2004; Norman 1999). Games with engaging stories and clever writing increase emotional impact (Isbister 2016) and players' sense of immersion during gameplay (Bissell 2010; Ermi \& Mäyrä 2005). Game studios that "have indulged the writing process," have created some of the most innovative and socially- engaged work to date (Bissell 2010). Creating inclusive content should be a consideration for all CGD educators.

The authors of trade press books on game writing define their field by juxtaposing it with common ideas about creative writing, likely driving the sentiment that "there is nothing like game writing." This sentiment is shared by some who teach creative writing in university. Kenneth Goldsmith (2011) asserted that the field of creative writing is stuck on perpetuating the incorrect notion of the original artist in writing. Goldsmith indicated that this attitude limits the potential of creative writing in the digital age. Others, like Mayers (2005), highlighted university creative writing programs' common refusal to theorize about the ways creative work operates. As a result, potentially valuable qualitative studies of these practices remain non-existent.

\section{CONCLUSION}

Basing curriculum design and instruction on research, rather than on generalized assumptions regarding industry trends and practitioners' anecdotes, adds value to the students' CGD education, which is vital to programs competing with the unbundled alternatives of online self-instruction in the 
technological tools of the trade (Selingo 2013). We have to teach students more than just technology; we have to ask ourselves, "What can we give students that the internet cannot?” Engaging our expertise as educators, amplified by research on practitioners in the field, will certainly present answers to this question.

Given the complexity of game development, complicated by poorly-defined roles and a lack of standard practices (Newman 2013), innovative research on industry practices, with the ultimate purpose of pedagogical application, is the key to advancing computer game design as a field of study. Any existing gaps in communication between the game industry and higher education should not serve as an excuse to retreat to our respective bases of understanding. Rather, those gaps are indicative of an opportunity for researchers to engage with a new field that represents a convergence of technology, art, storytelling, and interactivity in the digital age. Deployed in pedagogy, those efforts will certainly benefit the students we seek to serve and our field of study; they may also innovate the medium itself.

\section{BIBLIOGRAPHY}

Ashton, D. 2010. "Productive Passions and Everyday Pedagogies: Exploring the Industry-Ready Agenda in Higher Education.” Art, Design \& Communication in Higher Education, 9(1), 41-56. doi:10.1386/adch.9.1.41_1

Bateman, C. M. 2007. Game Writing: Narrative Skills for Videogames. Boston, MA: Charles River Media.

Bates, B. 2004. Game Design (2nd ed.). Boston, MA: Course Technology PTR.

Bazerman, C., \& Prior, P. A. 2009. What Writing Does and How It Does It: 
Introduction to Analyzing Texts and Textual Practices. Mahwah, NJ: Lawrence Erlbaum \& Associates.

Bissell, T. (2010). Extra Lives: Why Video Games Matter. New York, NY: Pantheon Books.

Brinkmann, S., \& Kvale, S. 2014. InterViews: Learning the Craft of Qualitative Research Interviewing (3rd ed.). Los Angeles, CA: SAGE Publications, Inc.

Brown, H. J. 2008. Videogames and Education. Retrieved from http://site.ebrary.com/lib/alltitles/

docDetail.action?docID=10292213

Chandler, R. 2007. Game Writing Handbook. Boston, MA: Cengage Learning.

Creswell, J. W. 2006. Qualitative inquiry and research design: Choosing among five approaches (2nd ed.). Thousand Oaks, CA: SAGE Publications, Inc.

Dansky, R. 2007. “Introduction to Game Narrative.” In Game Writing: Narrative Skills for Videogames, edited by C. M. Bateman, 1-23. Boston, MA: Cengage Learning.

De La Paz, S. \& McCutchen, D. 2011. "Learning to Write.” In Handbook of Research on Learning and Instruction, edited by R.E. Mayer and P.A. Alexander, 32-54. New York, NY: Routledge.

DeMarle, M. 2007. “Nonlinear Game Narrative.” In Game Writing: Narrative Skills for Videogames, edited by C. M. Bateman, 71-84. Boston, MA: Cengage Learning.

Despain, W. 2009. Writing for Video Game Genres: From FPS to $R P G$. Boca Raton, FL: CRC Press. 
DeVoss, D. N., Eidman-Aadahl, E., \& Hicks, T. 2010. Because digital writing matters: Improving student writing in online and multimedia environments. San Francisco, CA: Jossey-Bass.

Devitt, A. J. 2000. "Integrating Rhetorical and Literary Theories of Genre.” College English, 62(6), 696-718. https://doi.org/10.2307/ 379009

Dille, F., \& Zuur Platten, J. 2008. The Ultimate Guide to Video Game Writing and Design. New York, NY: Lone Eagle.

Egenfeldt-Nielsen, S., Smith, J. H., \& Tosca, S. P. 2015. Understanding video games: The essential introduction (3rd ed.). New York, NY: Routledge.

Engeström, Y. 1999. Communication, discourse and activity. The Communication Review, 3(1-2), 165-185. doi:10.1080/ 10714429909368577

Ermi, L., \& Mäyrä, F. 2005. "Fundamental Components of the Gameplay Experience: Analysing Immersion.” Worlds in Play: International Perspectives on Digital Games Research, 37(2), 37-53.

Foot, K. A. 2014. "Cultural-Historical Activity Theory: Exploring a Theory to Inform Practice and Research.” Journal of Human Behavior in the Social Environment, 24(3), 329-347. doi:10.1080/ 10911359.2013.831011

Goldsmith, K. 2011. Uncreative Writing: Managing Language in the Digital Age. New York, NY: Columbia University Press.

Heussner, T., Finley, T. K., Hepler, J. B., \& Lemay, A. 2015. The Game Narrative Toolbox. Burlington, MA: Focal Press.

Hudson, S. A. “Approaching a Pedagogy of Game Writing.” PhD diss., George Mason University, 2018. 
Hudson, S. \& Willis, B. 2019. “A Dancer and a Writer Walk Into a Classroom.” In The Power of Play in Higher Education: Creativity in Tertiary Learning, edited by A. James \& C. Nerantzi, 253-261. Basingstoke, England: Palgrave Macmillan.

Isbister, K. 2016. How Games Move Us: Emotion by Design. Cambridge, MA: MIT Press.

Jacobs, S. 2004. “Writesizing.” Game Developer, 11(10), 18-24.

Lebowitz, J., \& Klug, C. 2012. Interactive Storytelling for Video Games. Burlington, MA: Focal Press.

Maxwell, J. A. 2013. Qualitative Research Design: An Interactive Approach (3rd ed.). Thousand Oaks, CA: SAGE Publications, Inc.

Mayers, T. 2005. (Re)Writing Craft: Composition, Creative writing, and the Future of English Studies. Pittsburgh, PA: University of Pittsburgh Press.

Moustakas, C. 1994. Phenomenological research methods. doi:10.4135/9781412995658

Nardi, B. A. (1995). Context and consciousness: Activity theory and human-computer interaction. Cambridge, MA: MIT Press.

Newman, J. 2013. Videogames (2nd ed.). New York, NY: Routledge.

Norman, D. A. 1999. The Invisible Computer: Why Good Products Can Fail, the Personal Computer is so Complex, and Information Appliances Are the Solution. Cambridge, MA: MIT Press.

O’Donnell, C. 2014. Developer's dilemma: The secret world of videogame creators [Kindle version]. Retrieved from http://www.amazon.com 
Peery, J. 2016. “Game Writing in Practice-MMORPG Quests.” In Play/write: Digital Rhetoric, Writing, and Games, edited by D. Eyman and A. Davis, 347-362. Anderson, SC: Parlour Press

Prior, P. A. 2009. “Tracing process: How texts come into being.” In What Writing Does and How It Does It: Introduction to Analyzing Texts and Textual Practices, edited by C. Bazerman and P.A. Prior. Mahwah, NJ: Lawrence Erlbaum \& Associates.

Russell, D. R. 2010. "Writing in Multiple Contexts: Vygotskian CHAT Meets the Phenomenology of Genre." In Traditions of Writing Research edited by C. Bazerman, R. Krut, K. Lunsford, S. McLeod, S. Null, P. Rogers, and A. Stansell, 353-364. New York, NY: Routledge.

Russell, D.R. 2001. Where do the naturalistic studies of WAC/ WID point? A research review. In S.H. McLeod et al. (Eds.), WAC for the new millennium: strategies for continuing writing-acrossthe-curriculum-programs (pp. 259-298). Fort Collins, CO: WAC Clearinghouse.

Salmond, M. 2016. Video Game Design: Principles and Practices From the Ground Up. London, England: Fairchild Books.

Schön, D. 1983. The Reflective Practitioner: How Professionals Think in Action. New York, NY: Basic Books.

Selingo, J. 2013. College (Un)Bound: The Future of Higher Education and What It Means for Students. Boston: Houghton Mifflin Harcourt.

Sheldon, L. 2013. Character Development and Storytelling for Games (2nd ed.). Boston, MA: Course Technology.

Skolnick, E. 2014. Video Game Storytelling: What Every Developer Needs to Know About Narrative Techniques. Berkeley, CA: Watson-Guptill. 
116 Seth Andrew Hudson, PhD

Swales, J. 1990. Genre Analysis: English in Academic and Research Settings. Cambridge, England: Cambridge University Press.

Van Manen, M. 1990. Researching Lived Experience: Human Science for an Action Sensitive Pedagogy. Albany, NY: State University of New York Press.

Vygotsky, L. 1978. Mind in society: The development of higher psychological processes. Cambridge, MA: Harvard University Press.

Wengraf, T. 2001. Qualitative research interviewing. Thousand Oaks, CA: SAGE Publications, Ltd. doi:10.4135/9781849209717 